\title{
The electrical, optical, and structural properties of GaN epitaxial layers grown on $\mathrm{Si}(111)$ substrate with $\mathrm{SiN}_{x}$ interlayers
}

\author{
Engin Arslan a,*, Özgür Duygulu ${ }^{\text {b }}$, Ali Arslan Kaya ${ }^{c}$, Ali Teke ${ }^{\mathrm{d}}$, \\ Süleyman Özçelik ${ }^{\mathrm{e}}$, Ekmel Ozbay ${ }^{\mathrm{f}}$ \\ ${ }^{a}$ Nanotechnology Research Center, Bilkent University, Bilkent, 06800 Ankara, Turkey \\ ${ }^{\mathrm{b}}$ TUBITAK Marmara Research Center, Materials Institute, PO Box 21 Gebze Kocaeli, 41470, Kocaeli, Turkey \\ ${ }^{\mathrm{c}}$ Mugla University, Engineering Faculty, Metallurgy and Materials Engineering Department, 48170 Mugla, Turkey \\ ${ }^{\mathrm{d}}$ Department of Physics, Faculty of Science and Letters, Balikesir University, 10145 Balikesir, Turkey \\ ${ }^{\mathrm{e}}$ Department of Physics, Faculty of Science and Arts, Gazi University, Teknikokullar, 06500 Ankara, Turkey \\ ${ }^{\mathrm{f}}$ Nanotechnology Research Center, Department of Physics, Department of Electrical and Electronics Engineering, Bilkent University, \\ Bilkent, 06800 Ankara, Turkey
}

\section{A R T I C L E I N F O}

\section{Article history:}

Received 26 June 2009

Received in revised form

23 September 2009

Accepted 24 September 2009

Available online 12 October 2009

\section{Keywords:}

B1. GaN

B1. $\mathrm{SiN}_{x}$ layer

A3. MOCVD

B1. Silicon substrates

B1. Nitridation

\begin{abstract}
A B S T R A C T
The effect of the in situ substrate nitridation time on the electrical, structural and optical properties of GaN films grown on $\mathrm{Si}(111)$ substrates by metal organic chemical vapor deposition (MOCVD) was investigated. A thin buffer layer of silicon nitride $\left(\mathrm{SiN}_{x}\right)$ with various thicknesses was achieved through the nitridation of the substrate at different nitridation times ranging from 0 to $660 \mathrm{~s}$. The surface roughness of the GaN film, which was grown on the Si substrate $10 \mathrm{~s}$, exhibited a root mean square (RMS) value of 1.12 $\mathrm{nm}$ for the surface roughness. However, further increments in the nitridation times in turn cause increments in the surface roughness in the GaN layers. The number of threading dislocation (TD) was counted from plan-view TEM (Transmission Electron Microscopy) images. The determined density of these threading dislocations was of the order of $9 \times 10^{9} \mathrm{~cm}^{-2}$. The sheet resistances of the GaN layers were measured. The average sheet resistance significantly increases from $2867 \Omega \mathrm{sq}^{-1}$ for sample A (without nitridation) to $8124 \Omega \mathrm{sq}^{-1}$ for sample $\mathrm{F}$ (with $660 \mathrm{~s}$ nitridation). The photoluminescence (PL) measurements of the samples nitridated
\end{abstract}

\footnotetext{
* Corresponding author. Tel.: +90 312 2901971; fax: +90 3122901015.

E-mail address: engina@bilkent.edu.tr (E. Arslan).
} 
at various nitridation times were done at a temperature range of 10-300 K. A strong band edge PL emission line, which was centered at approx. $3.453 \mathrm{eV}$ along with its phonon replicas which was separated by approx. $92 \mathrm{meV}$ in successive orders, was observed at $10 \mathrm{~K}$. The full width at half maximum (FWHM) of this peak is approx. $14 \mathrm{meV}$, which indicates the reasonable optical quality of the GaN epilayers grown on Si substrate. At room temperature, the peak position and FWHM of this emission became $3.396 \mathrm{eV}$ and 58 $\mathrm{meV}$, respectively.

(c) 2009 Elsevier Ltd. All rights reserved.

\section{Introduction}

Because of the large direct energy band-gap and good thermal stability properties, GaN and related materials have attracted much attention due to their numerous optoelectronic and electronic applications, such as laser diodes (LDs), visible-blind ultraviolet (UV) detectors, and short-wave lightemitting diodes (LEDs) [1-4]. For the success of the operating devices, a high-quality material is essential. It is well known that the reliability and performance of these III nitride devices is very much dependent on the quality of the GaN epitaxial layers. One of the important issues in semiconductor technology is the availability of suitable substrates [5]. Many factors have to be considered for the selection of a suitable substrate, such as the lattice constants, thermal-expansion coefficients, crystal symmetry, chemical similarity with the epitaxial layer, costs, and available sizes. On the other hand, the growth of III nitrides is complicated because of the lack of lattice and thermal-expansion coefficient matched substrates. The lack of a lattice-matching substrate continues to be a challenge for the growth of the group III nitrides. The epitaxial growth of nitrides is commonly carried out on sapphire [5], SiC [6], Si [7], and ZnO. Due to its high crystal quality, low cost, good electrical and thermal conductivity, as well as large-area size as a substrate silicon is one of the most suitable alternative for the growth of GaN and AlGaN epitaxial layers [8,9].

Large mismatches in the lattice parameters $(-16.9 \%)$ and thermal-expansion coefficients (approx. 113\%) between the GaN and the Si bring out the problems in the growth of GaN films on a Si wafer such as cracking in the epitaxial layer [5,7,10-12]. Also large lattice mismatch causes high density of threading dislocations on the order of $\left(10^{9} \mathrm{~cm}^{-2}-10^{10} \mathrm{~cm}^{-2}\right)$ in the GaN film on silicon substrates, which significantly limits the performance of GaN based devices [8]. Different research groups have proposed various types of growth conditions and buffer layers as well as post-growth heat treatment processes in order to obtain crack-free high-quality GaN film on Si substrate [10-16]. But, the appearance of the cracks is quite random on the film, which produces significant difficulty in device applications. Because of these reasons, the control of crack distribution for a large-area film is the main goal of the present study $[7,9,10]$.

The high-quality GaN layers on Si substrates can only be achieved by an interlayer structure between the GaN and Si substrate. The only way to eliminate cracks is to control the stress in the interlayer. The defect-free GaN on Si growth were realized by using a $\mathrm{Si}_{x} \mathrm{~N}_{y}$ interlayer in hot wall chemical vapor deposition [13] via MOCVD [14] growth techniques. Huang et al. [13] achieved $\mathrm{Si}_{x} \mathrm{~N}_{y}$ interlayers by nitridating the Si substrate with $\mathrm{N}_{2}$ flow at $900{ }^{\circ} \mathrm{C}$ and Wu-Yih Uena et al. [14] achieved these interlayers at $1120{ }^{\circ} \mathrm{C}$. Wu-Yih Uena et al. [14] demonstrated that the nitridation temperature greatly affects optical properties and the surface morphology of the GaN films. With a similar nitridation process, a double-buffer structure of $\mathrm{AlN} / \mathrm{Si}_{x} \mathrm{~N}_{y}$ was used to obtain high-quality GaN film on Si substrate by using molecular beam epitaxy (MBE) [15]. The single-crystalline $\mathrm{SiN}_{x}$ was obtained by introducing the active nitrogen plasma to the $\mathrm{Si}(111)$ surface at $900{ }^{\circ} \mathrm{C}$ for approximately $30 \mathrm{~s}$ [15]. Also, Hageman et al. [16] reported significant improvements in the optical and structural properties of the $1 \mu \mathrm{m} \mathrm{GaN}$ layer with the creation of an $\operatorname{SiN}_{x}$ buffer layer.

In the present paper, we investigated the effects of the in situ substrate nitridation on the surface morphology by atomic force microscopy (AFM), structural properties by high resolution $\mathrm{x}-$ ray diffraction (HRXRD) and transmission electron microscopy (TEM), the electrical (sheet resistance) 
and optical properties (PL) of the hexagonal epitaxial GaN layers that were grown on $\mathrm{Si}(111)$ in the large nitridation time range. The in situ Si substrate nitridation processes were done in time range $0 \mathrm{~s}-660 \mathrm{~s}$.

\section{Experimental procedure}

GaN epitaxial layers on $\mathrm{Si}(111)$ substrate were grown in a low-pressure MOCVD reactor (Aixtron 200/4 HT-S). The reactant source materials for Ga, Al, and N were trimethylgallium (TMGa), trimethylaluminum (TMAl), and $\mathrm{NH}_{3}$, respectively. The $\mathrm{H}_{2}$ was used as a carrier gas during AlN and GaN growth. Before loading, the $\mathrm{Si}$ substrates were sequentially degreased by $\mathrm{H}_{2} \mathrm{SO}_{4}: \mathrm{H}_{2} \mathrm{O}_{2}: \mathrm{H}_{2} \mathrm{O}(2: 1: 1)$ solutions for $1 \mathrm{~min}$, and etched in a $2 \% \mathrm{HF}$ solution for $1 \mathrm{~min}$, rinsed in de-ionized water, and then dried with a nitrogen gun. At the beginning of the growth of AlN, the substrate was baked in $\mathrm{H}_{2}$ ambient at $1100^{\circ} \mathrm{C}$ for $10 \mathrm{~min}$ in order to remove the native oxide. To grow a $\mathrm{SiN}_{X}$ interlayer on a $\mathrm{Si}(111)$ substrate surface, following thermal etching, the substrate was nitridated by exposing it to the $\mathrm{NH}_{3}$ flow of $0.900 \mathrm{slm}$ at $1020^{\circ} \mathrm{C}$. Nitridation was performed at five different times. The nitridation times were: 0 (without nitridation), 10,60,120, 420, and $660 \mathrm{~s}$ for samples A, B, C, D, E, and F, respectively. After the nitridation, for all of the samples, we grew an approximately $150 \mathrm{~nm}$ high temperature $\left(1100^{\circ} \mathrm{C}\right)$ AlN (HT-AlN) buffer layer. In all of the samples, the $250 \mathrm{~nm}$ GaN layers were grown at $1050{ }^{\circ} \mathrm{C}$. For sample A, in order to prevent the growth of an amorphous $\mathrm{SiN}_{x}$ interlayer, the technique of the Al pre-covering process of Si substrate was applied before the growth of the AlN buffer.

The crystalline quality of the GaN layers was examined by high resolution x-ray diffraction (HRXRD). The X-ray diffraction was performed by using a Bruker D-8 high resolution diffractometer system, delivering CuK $\alpha 1$ (1.540 Å) radiation. TEM (Transmission Electron Microscopy) was used to show the microstructure, crystal structure, and dislocations of the $c$-plane GaN grown with an $\mathrm{SiN}_{x}$ interlayer. TEM examinations were conducted on the plan-view and cross-sectional samples. For the cross-sectional TEM investigations, all of the samples were prepared via the conventional sandwich technique. Gatan 691 Precision Ion Polishing System (PIPS) was used to prepare the thin foils. The specimens were examined by using a JEOL 2100 High Resolution Transmission Electron Microscope $\left(\mathrm{LaB}_{6}\right.$ filament) operated at $200 \mathrm{kV}$. Images were taken by using a Gatan Model 694 Slow Scan CCD Camera. A JEOL 31630 side entry double tilt holder was used.

The optical properties were investigated by photoluminescence (PL) measurements. The PL measurements were carried out with the samples placed in a close-cycled cryostat in a temperature range of $10 \mathrm{~K}-300 \mathrm{~K}$. As an excitation source, a $30 \mathrm{~mW}, 325 \mathrm{~nm}$ He-Cd laser was used. The luminescence was collected by suitable lenses and then dispersed with a $550 \mathrm{~mm}$ spectrometer and detected by CCD. The sheet resistance of the GaN/AlN/Si(111) structures were studied by a Lehighton sheet resistance mapper. The surface morphology was characterized by atomic force microscopy (AFM).

\section{Results and discussion}

Fig. 1 shows the $\omega-2 \theta$ scan X-ray diffraction (XRD) patterns of the GaN films grown on a nitridated $\mathrm{Si}(111)$ substrate for all samples. As shown in Fig. 1, the diffraction patterns only exhibited the dominant Wurtzite GaN crystalline (002) peaks, plus the (002) peaks from the AlN layer, and the (111) peaks from the Si substrate $[5,8,6,7]$. This indicates that the hexagonal structure of the GaN epilayer has its [001] direction set parallel to the [111] of the Si substrate. For sample D, the (002) reflections of Wurtzite GaN were clearly observed at $17.31^{\circ}$ and the (111) peak from Si substrate at $14.18^{\circ}$ and AlN layer at $18.12^{\circ}$, respectively.

The FWHM of the HRXRD rocking curves have been used to quantify crystalline imperfection, relatively. The rocking curves of symmetric planes normally response mosaic distortions but insensitive to the pure edge threading dislocation because of these planes are undistorted by them [10-18]. Thus the (002) plane rocking curves are sensitive only to the screw and mixedtype threading dislocations. However, the rocking curves of (121) asymmetry plane can be used to detect the crystalline distortions caused by all type threading dislocations including pure edge dislocations. As seen in Fig. 2, the crystalline quality of GaN epilayers grown on nitridated Si(111) substrate was determined by the HRXRD rocking curves FWHM ( $\omega$-scans) of (002) symmetry planes 


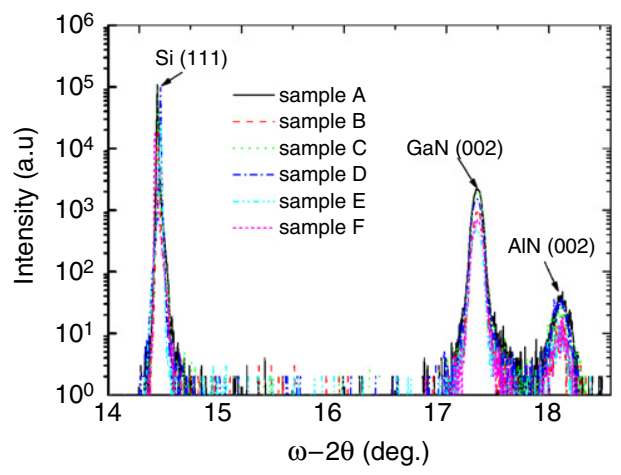

Fig. 1. The HRXRD $\omega-2 \theta$ scan pattern obtained from the GaN epilayer grown on the $\mathrm{Si}(111)$ substrate for all samples.

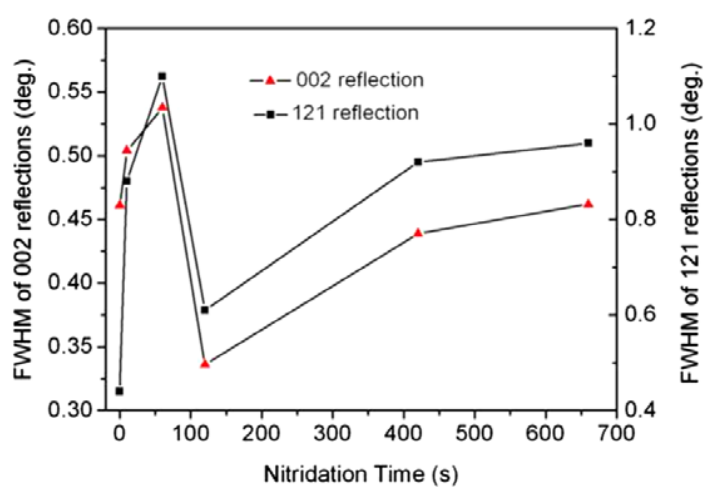

Fig. 2. The FWHM of the HRXRD rocking curve ( $\omega$-scans) of the (002) symmetry planes and (121) asymmetry planes of GaN epilayers as a function of nitridation times.

and (121) asymmetry planes of GaN epilayers. The FWHM of the GaN (002) peak were in the range of $0.336^{\circ}-0.538^{\circ}$. It is shown that with the nitridation times the FWHM of HRXRD peak increases from $0.461^{\circ}$ to $0.538^{\circ}$ as nitridation times increases from $0 \mathrm{~s}$ to $60 \mathrm{~s}$. respectively. On the other hand as nitridation times increased to $120 \mathrm{~s}$. the FWHM of the (002) peak was decreased to $0.336^{\circ}$. For more nitridation times, we obtained bigger values for FWHM of (002) symmetry planes of GaN layers. Also, we obtained similar behavior for FWHM of (121) asymmetry planes of GaN epilayers as a function of nitridation times.

The $2 \times 2 \mathrm{~mm}^{2}$ AFM images shown in Fig. 3(a)-(d) show a comparison of the surface morphology of the GaN epilayers with the substrates being nitridated for (a) without nitridation (sample A) (b) $60 \mathrm{~s}$ (sample C)(c) $120 \mathrm{~s}$ (sample D) (d) $660 \mathrm{~s}$ (sample F) at $1020^{\circ} \mathrm{C}$, respectively. The surface morphology of the GaN layer grown on the nitridated Si substrate is smooth. The root mean square (RMS) roughnesses are between $1.12 \mathrm{~nm}$ and $2.06 \mathrm{~nm}$. Fig. 4 shows the dependence of the RMS values of the GaN surface on the nitridation time. Sample B has a smooth surface with low RMS roughness ( $\mathrm{rms}=1.12 \mathrm{~nm}$ ) compared to samples A, C, D, E, and F. As can be seen, the root mean square (RMS) value of surface roughness decreases from $1.37 \mathrm{~nm}$ to $1.14 \mathrm{~nm}$ when the nitridation time was changed from 0 to $10 \mathrm{~s}$. However, further increments in the nitridation time in turn cause increments in the surface roughness. The RMS values increase to $1.98 \mathrm{~nm}$ when the nitridation time was set to $60 \mathrm{~s}$. Then, when the nitridation time was raised to 120 and a larger value, the surface becomes rough, as shown in Fig. 3 where the RMS value of the surface roughness increases to $2.06 \mathrm{~nm}$. Additionally, many dot-like crystallites are found on this rough surface. Therefore, the optimal nitridation time is $10 \mathrm{~s}$. 

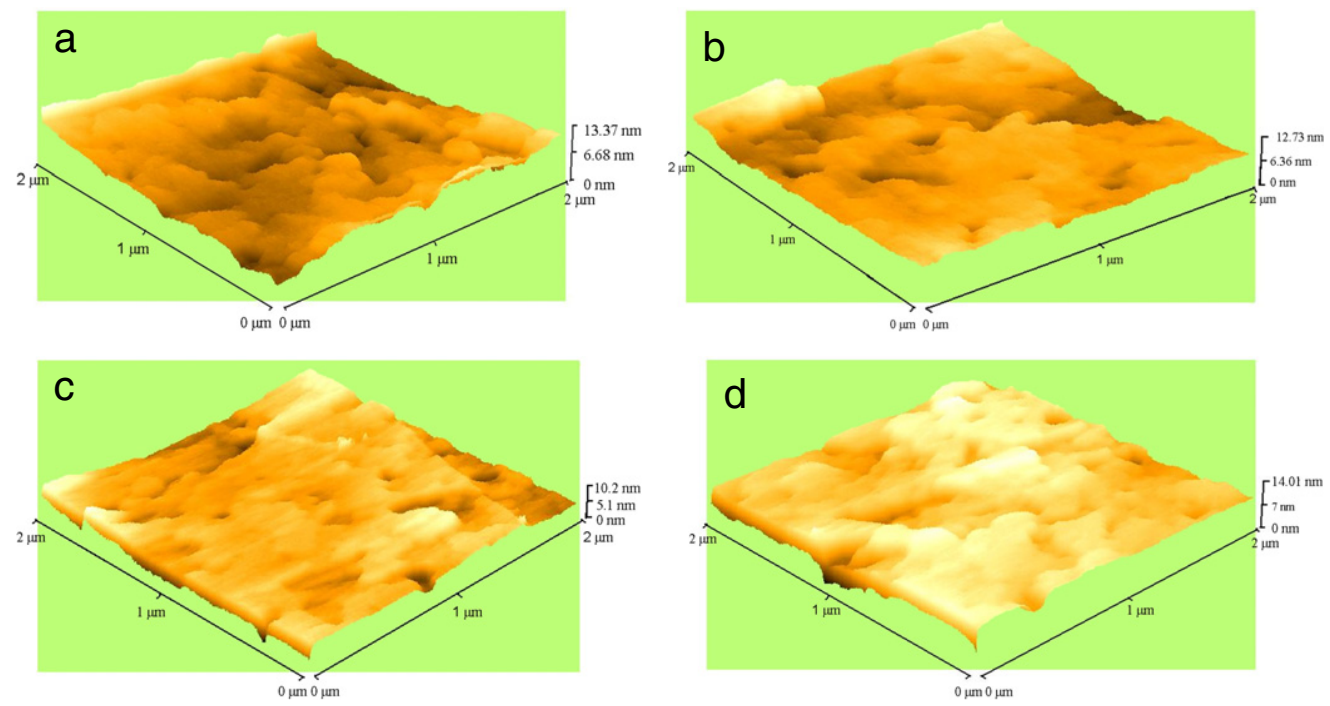

Fig. 3. The atomic force microscopy (AFM) image $\left(2 \times 2 \mathrm{~nm}^{2}\right)$ of GaN surfaces for, (a) sample A, (b) sample C, (c) sample D, and (d) sample F.

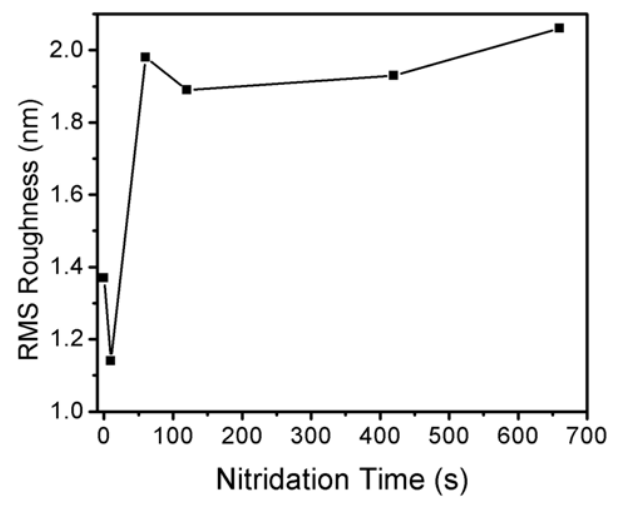

Fig. 4. The RMS roughness of the $\mathrm{GaN} / \mathrm{AlN} / \mathrm{SiN}_{x} / \mathrm{Si}$ (111) structures as a function of the nitridation time measured by AFM. The scanned area is $2 \times 2 \mathrm{~nm}^{2}$.

The dependence of the average sheet resistance of the GaN layers nitridation time is shown in Fig. 5. The average sheet resistance significantly increases from $2867 \Omega \mathrm{sq}^{-1}$ for sample A without nitridation to $8124 \Omega \mathrm{sq}^{-1}$ for sample F with $660 \mathrm{~s}$ nitridation time. For the GaN layers without nitridation (sample A) and a nitridation time of $10 \mathrm{~s}$ (sample B), a lower average sheet resistance of $2867 \Omega \mathrm{sq}^{-1}$ and $2868 \Omega \mathrm{sq}^{-1}$ were obtained, respectively. By increasing the nitridation time from 10 to $660 \mathrm{~s}$, the resistance increased to $8124 \Omega \mathrm{sq}^{-1}$ for sample $\mathrm{F}$, with a nitridation time of $660 \mathrm{~s}$. Fig. 6 shows the sheet resistance map of sample $\mathrm{C}$, with a nitridation time of $60 \mathrm{~s}$. The resistance map has a maximum resistance of $5562 \Omega \mathrm{sq}^{-1}$ and a minimum value of $4768 \Omega \mathrm{sq}^{-1}$. The average sheet resistance was $5178 \Omega \mathrm{sq}^{-1}$, with resistance uniformity that was equal to $96.5 \%$.

The microstructure of the GaN/AIN/SiN ${ }_{x}$ layers was investigated by using TEM. The cross-sectional TEM images of the GaN films on Si(111) substrate (a) without nitridation (sample A) and with (b) $60 \mathrm{~s}$ (sample C), (c) $120 \mathrm{~s}$ (sample D) and (d) $660 \mathrm{~s}$ (sample F) nitridated buffer layer, respectively, were shown in Fig. 7. The GaN/AIN layers and Si substrate were viewed in the [1 100$]$ and [11 $\overline{2}$ ], projections, 


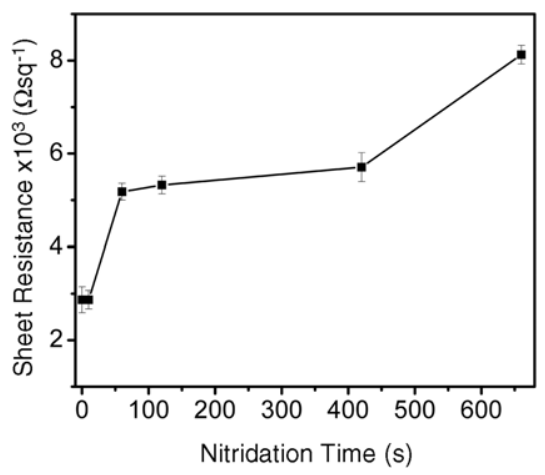

Fig. 5. Average sheet resistance of the $\mathrm{GaN} / \mathrm{AlN} / \mathrm{SiN}_{x} / \mathrm{Si}(111)$ structures as a function of the nitridation time. Error bars used for the standard deviation from 55 point map.

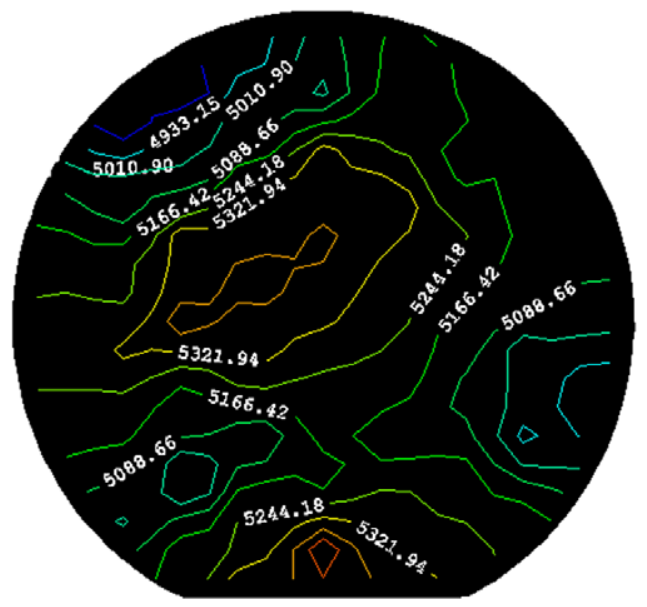

Fig. 6. Sheet resistance map of the 2 -inch GaN/AIN $/ \mathrm{SiN}_{x} / \mathrm{Si}$ (111) structure with a nitridation time of $60 \mathrm{~s}$ (sample C). The average sheet resistance and resistance uniformity is $5178 \Omega \mathrm{sq}^{-1}$ and $96.5 \%$, respectively.

respectively. The interfaces between the AlN nucleation layer and GaN films are relatively rough and high density of threading dislocations (TD) can be clearly observed.

As can be seen in Fig. 7 the TDs decreases rapidly within approx. $50 \mathrm{~nm}$ from the AlN/GaN interface into the GaN layer. Because of the dislocations can not end within a crystal structures, the ends of a dislocation can only be free at surfaces or at interfaces. The threading dislocations originating from the GaN/AlN interface may change direction and bend back to the interface. Tilt or twist boundaries that are said to exist within the GaN layer may also assist in such changes in dislocation directions [17].

The crystallographic relationship between the AlN layer and Si substrate, as well as the apparent stresses that, consequently, also leads to the observed high dislocation density within the AlN layer $[12,18,19]$. In the case of the interface was truly between the AlN layer and Si substrate, the crystallographic relationship and relatively high dislocation density, originating from the interface due to the large lattice mismatch between the two crystals, would provide a satisfactory explanation. However, in the present study and others, the AlN/Si interface contains an amorphous $\operatorname{SiN}_{x}$ layer [20-22,33].

The crystallographic relationship between AIN and Si has been addressed by many reports, in spite of the amorphous $\operatorname{SiN}_{x}$ between them. One of the proposals was that the amorphous $\operatorname{SiN}_{x}$ layer has some short-range order that necessitates the orientation of the AlN layer in this fashion [20]. According 


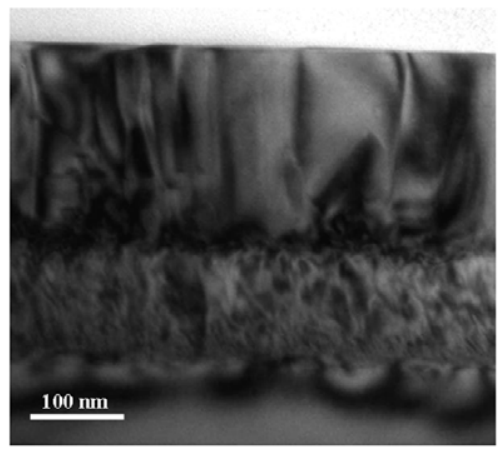

a

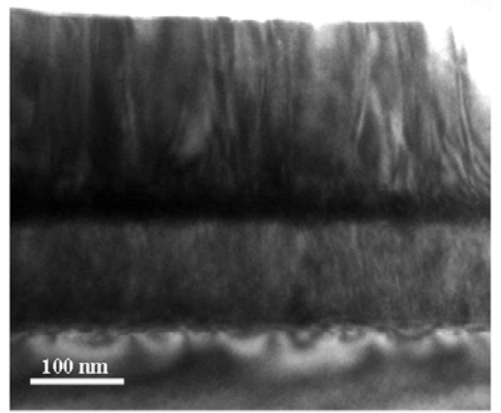

C

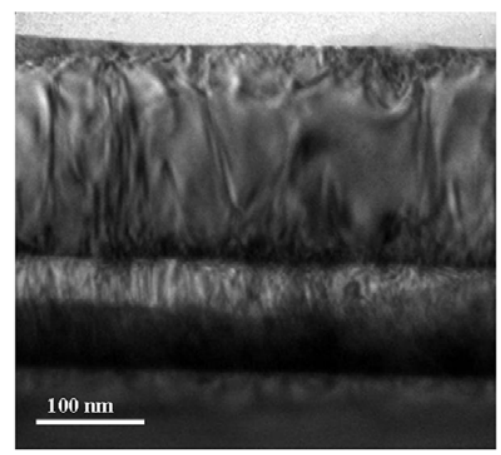

b

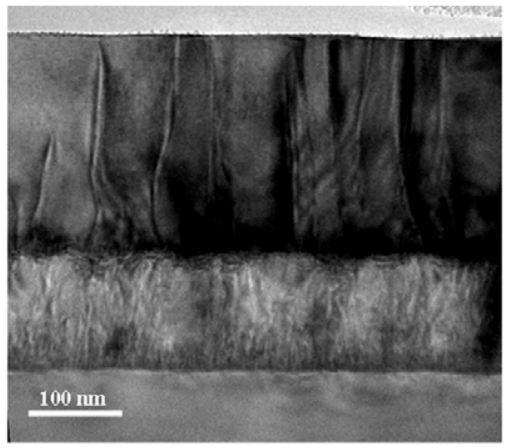

d

Fig. 7. Cross-sectional bright-field TEM image of (a) sample A, (b) sample C, (c) sample D, and (d) sample F.

to some, there exists in the $\operatorname{SiN}_{x}$ a top layer of a few atomic distances that is crystalline [21]. Kaiser et al. [20], Nakada et al. [21] and Dobos et al. [22] indicated that the epitaxial relationship is transferred from Si substrate into AlN and GaN layers through the amorphous $\operatorname{SiN}_{x}$ layer by the presence of crystalline regions, $\mathrm{Si}_{3} \mathrm{~N}_{4}$ inclusions, or holes in the $\mathrm{SiN}_{x}$ layer. Other explanations include the shortrange tetrahedral coordination that was maintained in the $\operatorname{SiN}_{x}$ layer [20], and the nucleation and growth of GaN islands from intrinsic or extrinsic defects by a "pinhole model" [23].

In our opinion, possibly the most reasonable explanation is that the amorphous $\mathrm{SiN}_{x}$ layer is not continuous and that there must be some points of actual contact between the crystalline Si substrate and deposited AlN layer [23]. Also, it may further be suggested that the tilt and twist boundaries that are said to exist in GaN may also be related to the growth modes of the AlN layer.

A plain AlN film grown on Si substrate contains a large number of threading dislocations [18]. Follstaedt et al. [19] and Datta et al. [24] have demonstrated that plan-view TEM imaging is generally accepted as a more trustworthy method for TD density measurement compared to other techniques, such as cathodoluminescence (CL) and atomic force microscopy (AFM). However, these techniques cannot be used effectively to detect a-type edge dislocations that thread to the film surface, since such dislocations do not create steps on the surface that are detectable by AFM, and are probably not effective nonradiative recombination centers that are detectable by CL $[19,24,25,20]$.

All of the plan-view images of the GaN on Si samples were bright-field (BF) images, which were collected by using multi-beam diffraction conditions and shown in Fig. 8 for (a) sample A, (b) sample C, (c) sample D, and (d) sample F. The plan-view specimens were investigated in the [0001] zoneaxis orientation, which were very close to the optical axis. Furthermore, the crystal defects, which were shown by cross-sectional studies, did not propagate to the surface, and cannot be seen in the plan-view images $[18,20,26]$. 


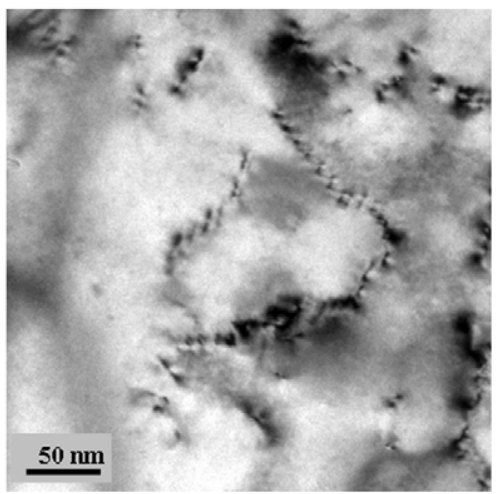

a

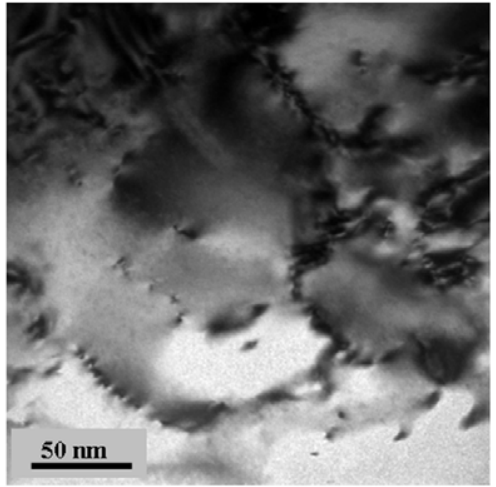

C

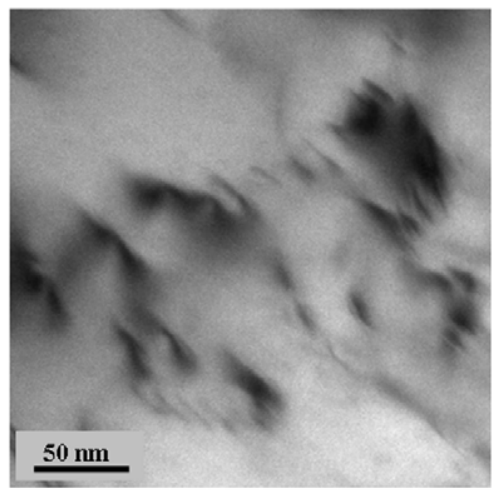

b

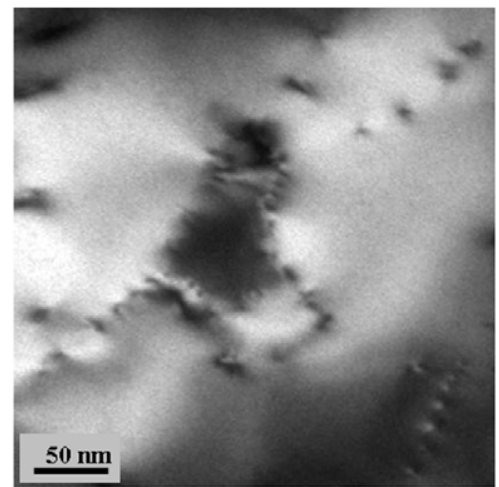

d

Fig. 8. Plan-view TEM micrographs of the GaN layer on the AlN nucleation overlayer grown with the insertion of an $\mathrm{SiN}_{x}$ layer for (a) sample A, (b) sample C, (c) sample D, and (d) sample F.

In Fig. 8, a network structure of defect arrays can be observed and the images show that the TDs in the epitaxial layers are not uniformly distributed on the film surfaces. The TDs can be determined from the plan-view TEM images [19,24]. In order to determine the TD densities, the number of TDs was counted for approximately 10 different plan-view TEM images which are similar to those shown in Fig. 8. All of the images were collected with their GaN [0001] zone axis for TD density determination. The determined density of these threading dislocations was order of $9 \times 10^{9} \mathrm{~cm}^{-2}$.

Fig. 9 shows the evolution of the PL spectra for sample D (120 s nitridation), which was chosen as an example, over a temperature range of $10 \mathrm{~K}-300 \mathrm{~K}$. As seen in this figure, the PL spectra at $10 \mathrm{~K}$ were dominated by a peak centered at $3.453 \mathrm{eV}$, in which its phonon replicas were separated by approx. $92 \mathrm{meV}$ in successive order. The full width at half maximum (FWHM) of this peak is approx. $14 \mathrm{meV}$. At room temperature, the peak position and FWHM of this emission became $3.396 \mathrm{eV}$ and $58 \mathrm{meV}$, respectively. The origin of this peak was attributed to the recombination of A-free exciton bound to the neutral shallow donor $\left(D^{0} X_{A}\right)$. These types of transitions are commonly responsible for the dominant PL line in the $n$-type GaN grown by any technique on any substrate. In high-quality strain-free GaN samples with a low concentration of defects, the $D^{0} X_{A}$ emission at $3.471 \mathrm{eV}$ sometimes comprises two or more sharp lines, tentatively attributed to different shallow donors $[27,28]$. In our case, the energy peak position of the $D^{0} X_{A}$ transition redshifted by nearly $18 \mathrm{meV}$ compared to the strain-free GaN template, which implies that there is a strong tensile stress that is present in the GaN epilayers grown on $\mathrm{Si}(111)$. The shoulder at $3.479 \mathrm{eV}$ that was observed at the high energy side of the spectrum was attributed to the A-free exciton transition [27,28]. Additional defects related 


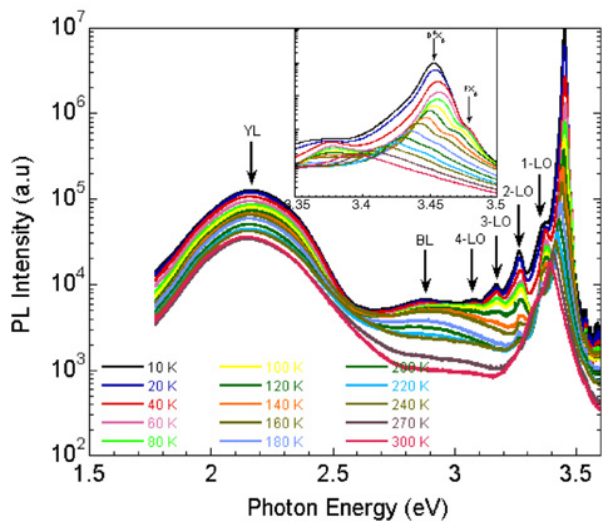

Fig. 9. The temperature dependence of the PL spectra and assignment of the peaks for sample D.

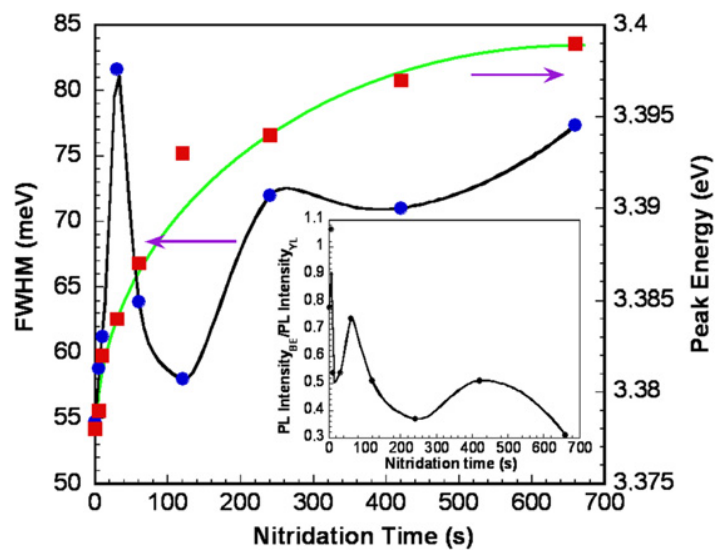

Fig. 10. Bound exciton $\left(D^{0} X_{A}\right)$ peak energy and its FWHM as a function of the nitridation time. The inset shows the integrated intensity ratio of $D^{0} X_{A}$ to $\mathrm{YL}$.

to weak blue luminescence (BL) and strong yellow luminescence (YL) peaks at $2.88 \mathrm{eV}$ and $2.16 \mathrm{eV}$, respectively, were also observed at the low energy side of the PL spectra at $10 \mathrm{~K}$. The YL is attributed to a shallow donor deep acceptor transition [29]. The deep acceptor is believed to be the native gallium vacancy $\left(\mathrm{V}_{\mathrm{Ga}}\right)$, while the shallow donor is attributed to substitutional oxygen $\left(\mathrm{O}_{\mathrm{N}}\right)$ and silicon $\left(\mathrm{Si}_{\mathrm{Ga}}\right)$ or $\mathrm{C}$ impurities substituted for the nearest neighbor of the $\mathrm{Ga}$ sites [30,31]. In our case, $\mathrm{V}_{\mathrm{Ga}}-\mathrm{Si}_{\mathrm{Ga}}$ complexes are believed to be the main cause of the YL due to the diffusion of Si impurities from the substrate during growth. The BL band peaking at approx. $2.9 \mathrm{eV}$ in $\mathrm{GaN}$ is similar to the notorious YL in this material. The BL band is often observed in undoped, $\mathrm{Mg}$ - and $\mathrm{Zn}$-doped GaN with a very similar shape and position [29].

Fig. 10 shows the PL peak energy and FWHM of band edge GaN emissions as a function of nitridation time. As seen in the figure, FWHM increases up to $82 \mathrm{meV}$ for the sample nitridated for $30 \mathrm{~s}$ and then decreases down to $58 \mathrm{meV}$ for the sample nitridated for $120 \mathrm{~s}$. It can be argued that the observed initial increase of the FWHM is probably due to increase in roughness of the surface of Si substrate which degraded the optical quality of the GaN epilayers. When the nitridation time increases further the growth of amorphous $\mathrm{SiN}_{x}$ layer serve as for the lateral growth of GaN epilayer. The PL FWHM of GaN epilayers was grown at the nitridation times longer than $120 \mathrm{~s}$ increases again. In the inset of Fig. 10 the peak intensity ratio of the donor-bound exciton $\left(D^{0} X_{A}\right)$ to yellow luminescence $(\mathrm{YL})$ is also shown for the samples grown at various nitridation times. There was no clear correlation observed 


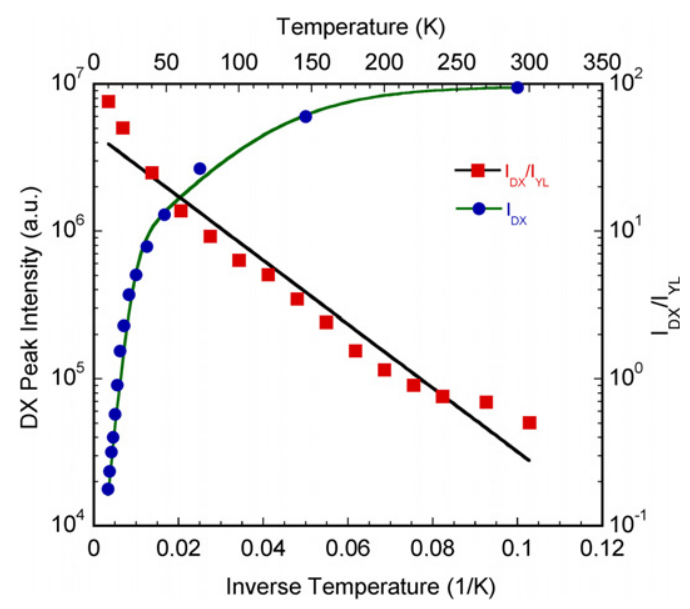

Fig. 11. $D^{0} X_{A}$ related PL intensity as a function of an inverse temperature and the temperature dependence of the relative intensities $\left(I_{\mathrm{DX}} / I_{\mathrm{YL}}\right)$ for sample $\mathrm{D}$. The solid line is drawn to calculate the activation energies by using Eq. (1). The black solid line represents the exponential fit to the temperature behavior of the $I_{\mathrm{DX}} / I_{\mathrm{YL}}$ ratio.

between the relative ratio of the PL peak intensities and FWHM of band edge emission and nitridation time. The nitridation time dependence of the FWHM and the ratios of peak intensity of band edge to yellow luminescence emission show almost completely opposite characteristic. When the FWHM become smaller, indicating better optical quality of the sample the peak intensity ratio of band edge emission to yellow luminescence is worsen. This could not be explained explicitly, but it can be speculated that the peak intensity ratio decreases with an increasing nitridation time, due to probably enhanced Si diffusion into the GaN epilayers as the nitridation time becomes longer $[13,14,31]$. This behavior is opposite to the results of Huang et al. [13], and Wu-Yih Uena et al. [14] who reported a complete diminishing of the yellow luminescence band when the substrate nitridated before GaN growth. However, if one can ignore the observed unclear dependence on nitridation time, it would be concluded that the optical quality of the GaN epilayers grown on Si substrate become worsen as the nitridation time increase over $100 \mathrm{~s}$. In the same figure, the peak energy increases monotonically as the nitridation time increases which indicate the relaxation of tensile strain in GaN epilayers.

Fig. 11 shows an Arrhenius plot of the PL intensity for $D^{0} X_{A}$ over the temperature ranges under investigation. As shown in the figure, the PL intensity of the emission decreases as the temperature increases. The dominant mechanism leading to this thermal quenching of PL intensity is due to the increase in the effects of nonradiative recombination centers with temperature. As seen in the figure, the PL intensity of all three samples decreases rather little at low temperatures but decreases more rapidly at high temperatures. This suggests that there are two different mechanisms that are responsible at low and high temperature regimes. The activation energies in these thermally activated processes were calculated using the following equation [32],

$$
I(T)=\frac{I_{0}}{1+\sum_{i} \alpha_{i} \exp \left(-\frac{E_{a i}}{k_{B} T}\right)}
$$

where $E_{a i}$ is the activation energy of the corresponding nonradiative recombination centers and $\alpha_{i}$ is the process rate parameter. The fitted curve is shown as a solid line in Fig. 11. The thermal activation energies of $6 \mathrm{meV}$ at a low temperature and $56 \mathrm{meV}$ at high temperature regimes were deduced from the best fit by considering the biexponential function. At a low temperature, the thermal quenching of the PL peak intensity with $6 \mathrm{meV}$ activation energy could be related to the thermal dissociation of the main donor-bound exciton line at $3.453 \mathrm{~nm}$ into other shallower donors as discussed in the temperature dependence of the emission peak. The faster decreasing rate of PL intensity with $56 \mathrm{meV}$ thermal activation energy is related to an increase in nonradioactive recombination centers as the 
temperature increases. The temperature dependence of the relative intensities of the A-exciton bound to the neutral donor with respect to yellow luminescence $\left(I_{\mathrm{DX}} / I_{\mathrm{YL}}\right)$ is also shown in Fig. 11. This ratio decreases nearly exponentially with the temperature indicating that the defect density in this sample is high, which is probably due to the intensive diffusion of Si impurities from the Si substrate to the GaN epilayer.

\section{Conclusions}

We investigated the influence of the in situ substrate nitridation time on the electrical and optical properties of the hexagonal epitaxial GaN films grown on $\mathrm{Si}(111)$. The nitridation time lengths ranged from 0 to $660 \mathrm{~s}$. The nitridation processes were performed at $1020^{\circ} \mathrm{C}$. The surface of the $\mathrm{GaN}$ films was characterized with AFM. The surface roughness of the GaN film that was grown on the $10 \mathrm{~s}$ nitridated Si substrate exhibited a root mean square (RMS) value of $1.12 \mathrm{~nm}$ for the surface roughness. However, further increments in the nitridation times resulted in larger surface roughness in the GaN layers. The average sheet resistance significantly increased from $2867 \Omega \mathrm{sq}^{-1}$ for sample $\mathrm{A}$ (without nitridation) to $8124 \Omega \mathrm{sq}^{-1}$ for sample $\mathrm{F}$ (with $660 \mathrm{~s}$ nitridation). The structural characteristics, such as dislocation densities, were all studied in detail by using a planar and cross-sectional view of transmission electron microscopy (TEM). It was found that the dislocation density in GaN films was order of $9 \times 10^{9} \mathrm{~cm}^{-2}$.

The optical properties of the nitridated samples were investigated at a temperature range of $10 \mathrm{~K}$ to $300 \mathrm{~K}$. A strong band edge PL emission line centered at $3.453 \mathrm{eV}$ along with its phonon replicas separated by $\sim 92 \mathrm{meV}$ in successive orders were observed at $10 \mathrm{~K}$. The full width at half maximum (FWHM) of this peak is $\sim 14 \mathrm{meV}$ in turn indicating the reasonable optical quality of the GaN epilayers grown on Si substrate. At room temperature, the peak position and FWHM of this emission became $3.396 \mathrm{eV}$ and $58 \mathrm{meV}$, respectively. We measured the peak intensity ratio of a donor-bound exciton to yellow luminescence for the samples that were grown at various nitridation times. There was no clear correlation between FWHM of the GaN band edge emission, and the relative ratio of the PL peak intensities and nitridation times. However, it could be argued that the overall optical quality of the GaN epilayers become poorer as the nitridation time increases over $100 \mathrm{~s}$.

\section{Acknowledgements}

This work is supported by the European Union under the projects EU-METAMORPHOSE, EUPHOREMOST, EU-PHOME, and EU-ECONAM, and TUBITAK under the Project Numbers 105E066, 105A005, 106E198, and 106A017. One of the authors (E.O.) also acknowledges partial support from the Turkish Academy of Sciences.

\section{References}

[1] L. Shen, S. Heikman, B. Moran, R. Coffie, N.-Q. Zhang, D. Buttari, I.P. Smorchkova, S. Keller, S.P. DenBaars, U.K. Mishra, IEEE Electron Device Lett. 22 (2001) 457.

[2] A. Chini, D. Buttari, R. Coffie, L. Shen, S. Heikman, A. Chakraborty, S. Keller, U.K. Mishra, IEEE Electron Device Lett. 25 (2004) 229.

[3] Bayram Butun, Jean Cesario, Stefan Enoch, Romain Quidant, Ekmel Ozbay, Photonics Nanostruct. 5 (2007) 86.

[4] Serkan Butun, Mutlu Gokkavas, HongBo Yu, Ekmel Ozbay, Appl. Phys. Lett. 89 (2006) 073503.

[5] Engin Arslan, Serkan Bütün, S. Bora Lisesivdin, Mehmet Kasap, Suleyman Ozcelik, Ekmel Ozbay, J. Appl. Phys. 103 (2008) 103701.

[6] R. Gaska, J.W. Yang, A. Osinsky, Q. Chen, M. Asif Khan, A.O. Orlov, G.L. Snider, M.S. Shur, Appl. Phys. Lett. 72 (1998) 707.

[7] A. Dadgar, C. Hums, A. Diez, J. Bläsing, A. Krost, J. Cryst. Growth 297 (2006) 279.

[8] S. Pal, C. Jacob, Bull. Mater. Sci. 27 (2004) 501.

[9] Engin Arslan, Mustafa K. Ozturk, Ali Teke, Suleyman Ozcelik, Ekmel Ozbay, J. Phys. D: Appl. Phys. 41 (2008) 155317.

[10] Min-Ho Kim, Young-Gu Do, Hyon Chol Kang, Do Young Noh, Seong-Ju Park, Appl. Phys. Lett. 79 (2001) 2713.

[11] Kai Cheng, M. Leys, S. Degroote, B. Van Daele, S. Boeykens, J. Derluyn, M. Germain, G. Van Tendeloo, J. Engelen, G. Borghs, J. Electron. Mater. 35 (2006) 592.

[12] S. Raghavan, J.M. Redwing, J. Appl. Phys. 98 (2005) 023514.

[13] J. Huang, Z. Ye, L. Wang, J. Yuan, B. Zhao, H. Lu, Solid-State Electron. 46 (2002) 1231.

[14] Wu-Yih Uena, Zhen-Yu Li, Shan-Ming Lan, Sen-Mao Liao, J. Cryst. Growth 280 (2005) 335.

[15] C.-L. Wu, J.-C. Wang, M.-H. Chan, T.T. Chen, S. Gwo, Appl. Phys. Lett. 83 (2003) 4530.

[16] P.R. Hageman, S. Haffouz, V. Kirilyuk, A. Grzegorczyk, P.K. Larsen, Phys. Status Solidi A 188 (2001) 523. 
[17] T. Metzger, R. Höppler, E. Born, O. Ambacher, M. Stutzmann, R. Stömmer, M. Schuster, H. Göbel, S. Christiansen, M. Albrecht, H.P. Strunk, Phil. Mag. A 77 (1998) 1013.

[18] H. Lahreche, P. Vennegues, B. Beaumont, P. Gibart, J. Cryst. Growth 205 (1999) 245.

[19] D.M. Follstaedt, P.P. Provencio, N.A. Missert, C.C. Mitchell, D.D. Koleske, A.A. Allerman, C.I.H. Ashby, Appl. Phys. Lett. 81 (2002) 2758

[20] S. Kaiser, M. Jakob, J. Zweck, W. Gebhardt, O. Ambacher, R. Dimitrov, A.T. Schremer, J.A. Smart, J.R. Shealy, J. Vac. Sci. Technol. B 18 (2) (2000) 733.

[21] Yoshinobu Nakada, Igor Aksenov, Hajime Okumura, Appl. Phys. Lett. 73 (1998) 6951.

[22] L. Dobos, B. Pecz, E. Feltin, B. Beaumont, P. Gibart, Vacuum 71 (2003) 285.

[23] Masao Tamura, Máximo López-López, Tokuo Yodo, Superficies y Vacío 13 (2001) 80.

[24] R. Datta, M.J. Kappers, J.S. Barnard, C.J. Humphreys, Appl. Phys. Lett. 85 (2004) 3411.

[25] X. Weng, S. Raghavan, J.D. Acord, A. Jain, E.C. Dickey, J.M. Redwing, J. Cryst. Growth 300 (2007) 217.

[26] F. Wu, S. Zamir, B. Meyler, J. Salzman, Y. Golan, J. Electron. Mater. 31 (2002) 88.

[27] M.A. Reshchikov, D. Huang, F. Yun, L. He, H. Morkoç, D.C. Reynolds, S.S. Park, K.Y. Lee, Appl. Phys. Lett. 79 (2001) 3779.

[28] J.A. Freitas Jr., W.J. Moore, B.V. Shanabrook, G.C.B. Braga, S.K. Lee, S.S. Park, J.Y. Han, Phys. Rev. B 66 (2002) 233311.

[29] M.A. Reshchikov, H. Morkoç, J. Appl. Phys. 97 (2005) 061301.

[30] J. Neugebauer, C.G. Vande Walle, Appl. Phys. Lett. 69 (1996) 503.

[31] T. Mattila, R.M. Nieminen, Phys. Rev. B 55 (1997) 9571.

[32] P.J. Dean, Phys. Rev. 157 (1967) 655.

[33] Engin Arslan, Mustafa K. Öztürk, Özgür Duygulu, Ali Arslan Kaya, Süleyman Özçelik, Ekmel Özbay, Appl. Phys. A 94 (2009) 73. 